\title{
Stacking-Sequence Optimization of Composite Delta Wing to Improve Flutter Limit Using Fractal Branch and Bound Method*
}

\author{
Yoshiyasu HIRANO ${ }^{* *}$ and Akira TODOROKI***
}

\begin{abstract}
The authors developed a fractal branch and bound method for optimization of stacking sequences of laminated composites to maximize buckling load of composite structures. The method demands an approximation of a design space with a response surface comprising quadratic polynomials for pruning branches of stacking sequences. Approximation of the objective function with quadratic polynomials has been confirmed for buckling load maximizations and panel-flutter-speed-limit maximizations using lamination parameters as predictors. Flutter speed maximization of composite delta wing is employed in this study as an example of stacking-sequence optimization using the fractal branch and bound method. This paper describes the theoretical background of the fractal branch and bound method. Then approximations are performed using quadratic polynomials with lamination parameters as predictors. Subsequently, we investigated effectiveness of this method for supersonic flutter of a composite delta wing. Results indicate that the method was applied successfully; a practical optimal stacking-sequence was obtained using modified response surfaces.
\end{abstract}

Key Words: Optimum Design, Composite Material, Flutter, Response Surface, StackingSequence

\section{Introduction}

Many composite structures are adopted to reduce weight and improve aeroelastic responses for nextgeneration supersonic transportation aircraft. Composite laminates made from unidirectional prepreg sheets are employed for these composite structures because of their superior mechanical performance. For a laminated composite, it is indispensable to optimize stacking sequences of the laminated composites. Miki ${ }^{(1)}$ and Fukunaga ${ }^{(2)}$ proposed a graphical stacking-sequence optimization technique with lamination parameters.

For practical laminated composite structures, available fiber angles are quite limited to a small set of fiber angles such as $0^{\circ}, \pm 45^{\circ}$, and $90^{\circ}$ plies because of the shortage of various required experimental data for design and a

* Received 12th April, 2004 (No. 04-4093)

** Advanced Composite Evaluation Technology Center, Institute of Space Technology and Aeronautics, Japan Aerospace Exploration Agency, 6-13-1 Oosawa, Mitakashi, Tokyo 181-0015, Japan.

E-mail: hirano@chofu.jaxa.jp

*** Department of Mechanical Science and Engineering, Tokyo Institute of Technology, 2-12-1-I1-58 Ohokayama, Meguro-ku, Tokyo 152-8552, Japan hand lay-up processing method. A stacking-sequence optimization problem becomes a combinatorial optimization problem with some constraints because there are several constraints on practical stacking sequences.

The author's group has proposed a stacking-sequence optimization method with a genetic algorithm ${ }^{(3)-(5)}$. A new technique to reduce evaluation cost with response surface approximations in lamination parameters was employed because the genetic algorithm requires high computational cost as a result of the large number of evaluations of individuals ${ }^{(6)-(8)}$. These studies revealed that approximations of the objective function with a quadratic polynomial are applicable for buckling load maximization problems in the lamination parameters.

However, genetic algorithms constitute a probabilistic approach; they do not always give a true optimal result. This problem was solved using a newly proposed fractal branch and bound method ${ }^{(9)}$. The method requires approximation of the objective function in lamination parameters with a quadratic polynomial for trimming useless branches. This method was applied to a buckling load maximization problem. Its efficiency was confirmed in our previous paper ${ }^{(9)}$.

The flutter problem encountered by SSTs, a coupled problem of fluid dynamics and structural analyses, re- 
quires larger computational cost than the buckling problem of a laminate. The flutter problem usually has a sharp change of flutter speed limit; that sharp change is caused by changes of vibration modes ${ }^{(10)}$. This sharp change may produce multiple local maxima ${ }^{(11)}$. The possibility of local maxima compels us to investigate the applicability of a response surface of a quadratic polynomial: this is required to employ the fractal branch and bound method for optimization of the stacking-sequence of the panel flutter problem. Our previous study ${ }^{(12)}$ proposed a modified response surface. The method was confirmed for the panel flutter problem in supersonic flow. The panel flutter problem can be solved analytically; the problem does not require FEM analyses.

For practical design of a laminated composite wing, the flutter problem of an entire wing is more critical than the panel flutter problem, but flutter analyses require an FEM code of multidisciplinary analyses. The present study specifically addresses the maximization problem of a flutter speed limit of the composite delta wing. A multidisciplinary FEM code is developed here. Regarding the flutter problem, although lamination parameters are adopted as variables, the flutter problem provides a complicated objective function. It is required to confirm the applicability of the approximation of the response surface with a quadratic polynomial.

\section{Optimization Problem}

Flutter speed limit of a rigidly mounted thin, flat laminated delta wing is addressed in this study (Fig. 1). In Fig. 1, $a, b$, and $h$ represent span, chord length of the root, and thickness of a wing, respectively. The material properties are: T800/3 630; $E_{\mathrm{L}}=142 \mathrm{GPa} ; E_{\mathrm{T}}=10.8 \mathrm{GPa}$; $G_{\mathrm{LT}}=5.49 \mathrm{GPa} ; v_{\mathrm{LT}}=0.3 ; \rho=1.5 \times 10^{3} \mathrm{~kg} / \mathrm{m}^{3}$. The surface of this laminated delta wing is exposed in the steady supersonic flow $(\boldsymbol{V})$. When supersonic flow is steady, the flow can be approximated as a potential flow with an approximation of small oscillations from the steady flow. The following equation can represent the dynamic equation of

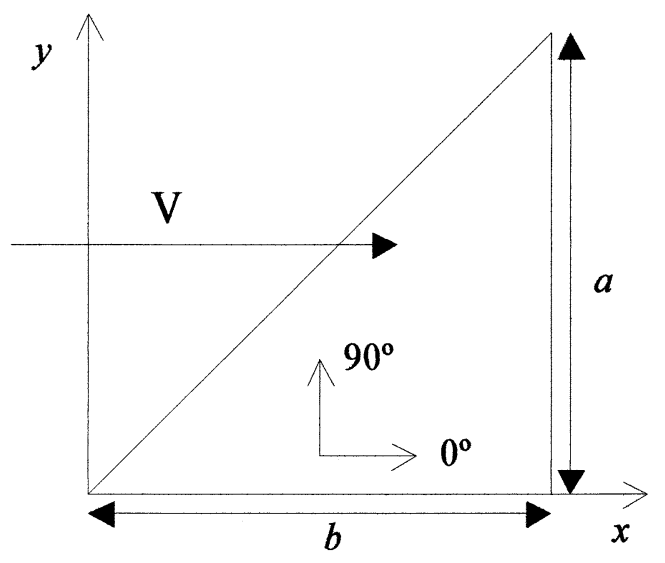

Fig. 1 Coordinate of delta wing and supersonic flow the wing. In this equation, only the deviation of the flow velocity from the steady flow is considered to affect wing vibration.

$$
\begin{aligned}
& D_{11} \frac{\partial^{4} w}{\partial x^{4}}+2\left(D_{12}+2 D_{66}\right) \frac{\partial^{4} w}{\partial x^{2} \partial y^{2}}+D_{22} \frac{\partial^{4} w}{\partial y^{4}} \\
& =-\rho_{\mathrm{L}} h \frac{\partial^{2} w}{\partial t^{2}}+p(x, y, t)
\end{aligned}
$$

In that equation, $w(x, y, z), \rho_{\mathrm{L}}$, and $h$ are displacement of the wing in the $z$-axis, density of the panel, and the thickness, respectively. A bending stiffness matrix comprise six elements; $D_{11}, D_{12}, D_{22}, D_{13}, D_{23}$, and $D_{66}$ are explained in detail in Ref. (13). In this study, $D_{13}$ and $D_{23}$ are neglected because these elements $D_{13}$ and $D_{23}$ are relatively smaller than other elements $\left(D_{11}, D_{12}, D_{22}\right.$, and $\left.D_{66}\right)$ for balanced symmetric laminates which comprise plies of $0^{\circ}$, $\pm 45^{\circ}$, and $90^{\circ}$. Minor deviation of pressure caused by the small deviation of supersonic flow $(\boldsymbol{V})$ from steady flow is expressed as $p(x, y, t)$; this pressure can be derived from Ref. (14) as the following.

$$
p(x, y, t)=-\left(\lambda \frac{\partial w}{\partial x}+\mu \frac{\partial w}{\partial t}\right)
$$

In Eq. (2), $\lambda$ and $\mu$ represent the dynamic pressure parameter and aerodynamic damping coefficient, respectively.

$$
\lambda=-\frac{4 q}{\sqrt{M^{2}-1}}, \quad \mu=\frac{\lambda\left(M^{2}-2\right)}{V\left(M^{2}-1\right)}
$$

In Eq. (3), $q$ and $M$ are the dynamic pressure of the steady flow and its Mach number, respectively.

In this study, a flutter speed limit is analyzed by means of a finite element method using non-conforming triangular elements ${ }^{(15)}$. The displacement field inside each element $w$ is represented as $w=[N]\left\{w_{e}\right\}$, where $[N]$ and $\left\{w_{e}\right\}$ are shape functions of a non-conforming triangular element and displacement of node point of an element. Virtual work by aerodynamic force $W_{p}$ is calculated as the following equation.

$$
\begin{aligned}
W_{p} & =\int_{A} p \bar{w} d A \\
& =-\left\{\bar{w}_{e}\right\}^{T}\left(\lambda \int_{A}[N]^{T}\left[N_{x}\right] d A\left\{w_{e}\right\}\right. \\
& \left.+\mu \int_{A}[N]^{T}[N] d A\left\{\dot{w}_{e}\right\}\right) \\
& =-\left\{\bar{w}_{e}\right\}^{T}\left[\beta\left[A_{e}\right]\left\{w_{e}\right\}+\mu\left[A d_{e}\right]\left\{\dot{w}_{e}\right\}\right] \\
& =-\left\{\bar{w}_{e}\right\}^{T}\{Q\}
\end{aligned}
$$

In Eq. (4), $\left[A_{e}\right]$ and $\left[A d_{e}\right]$ are the aerodynamic matrix and aerodynamic damping matrix for an element, respectively.

$$
\left\{\begin{array}{l}
{\left[A_{e}\right]=\int[N]^{T}\left[N_{x}\right] d A, \quad\left[A d_{e}\right]=\int[N]^{T}[N] d A} \\
\{Q\}=\lambda\left[A_{e}\right]\left\{w_{e}\right\}+\mu\left[A d_{e}\right]\left\{\dot{w}_{e}\right\}
\end{array}\right.
$$

Lagrange's equation of a typical element is shown as 


$$
\frac{d}{d t}\left(\frac{\partial T}{\partial \dot{w}_{i}}\right)+\frac{\partial U}{\partial w_{i}}=Q_{i},
$$

where $U$ is the energy of strain and $T$ is the energy of movement, and

$$
U=\frac{1}{2}\left\{w_{e}\right\}^{T}\left[k_{e}\right]\left\{w_{e}\right\}, \quad T=\frac{1}{2}\left\{\dot{w}_{e}\right\}^{T}\left[m_{e}\right]\left\{\dot{w}_{e}\right\},
$$

where $\left[k_{e}\right]$ and $\left[m_{e}\right]$ are the stiffness matrix and mass matrix for an element, respectively.

Substituting Eqs. (4), (5), and (7) into Eq. (6), the equation is organized as

$$
[M]\{\ddot{w}\}+[K]\{w\}+\lambda[A]\{w\}+\frac{\mu}{\rho h}[M]\{\dot{w}\}=0,
$$

where $\{w\}$ is defined as the node point displacement for the entire wing. In addition, $[M],[K]$ and $[A]$ are defined as the global mass matrix, global stiffness matrix, and global aerodynamic matrix, respectively.

Considering harmonic oscillation, node point displacement for the entire wing is assumed as $\{w\}=\{W\} e^{i \omega t}$. Equation (8) yields a complex eigenvalue problem as

$$
\{[K]+\lambda[A]\}\{W\}=\kappa[M]\{W\},
$$

where the global aerodynamic matrix $[A]$ is a nonsymmetric matrix. Equation (9), therefore, is a complex eigenvalue problem. In this study, QR algorithm ${ }^{(16)}$ is adopted to solve this complex eigenvalue problem.

Supersonic flow is assumed to be steady in this study. Therefore, a term of first order derivatives with respect to time can be neglected. The neglected term is aerodynamic damping here.

Eigenvalues are calculated by changing the Mach number $M$, i.e. $\lambda$. Thereby, the imaginary and real parts of the eigenvalues are obtained. Flutter limit $\lambda_{\mathrm{c}}$ is the point at which the two different eigenvalue modes coincide with each other first. The obtained flutter limit is normalized as

$$
\lambda_{\mathrm{c}}^{*}=\frac{a^{3}}{D_{0}} \lambda_{\mathrm{c}},
$$

where $D_{0}$ is the bending stiffness $D_{11}$ when a laminate comprises only $0^{\circ}$-plies; $\left(W_{1}^{*}, W_{2}^{*}\right)=(1.0,1.0)$.

Change of stacking-sequence of the laminate induces change of bending stiffness in Eq. (1). That causes change of flutter limit $\lambda_{\mathrm{c}}^{*}$.

\section{Optimization Method}

\section{1 Bending lamination parameters}

A symmetric laminate of the total number of plies $2 N s$ is considered here. Bending lamination parameters $\left(W_{1}^{*}, W_{2}^{*}\right)$ of a laminate are defined as

$$
\begin{aligned}
\boldsymbol{W} & =\left[\begin{array}{l}
W_{1}^{*} \\
W_{2}^{*}
\end{array}\right] \\
& =\frac{3}{h^{3} N s^{3}} \sum_{k=1}^{N s}\left\{(N s-k+1)^{3}-(N s-k)^{3}\right\}\left[\begin{array}{l}
\cos 2 \theta_{k} \\
\cos 4 \theta_{k}
\end{array}\right],
\end{aligned}
$$

where $k$ is the ply number counted from the outermost ply, $\theta_{k}$ is the fiber angle of the $k$ th ply, and $h$ is the total thickness of the laminate. Using lamination parameters, bending stiffness elements are expressed simply as

$$
\left[\begin{array}{c}
D_{11} \\
D_{22} \\
D_{12} \\
D_{66}
\end{array}\right]=\frac{h^{3}}{12}\left[\begin{array}{ccc}
U_{1} & W_{1}^{*} & W_{2}^{*} \\
U_{1} & -W_{1}^{*} & W_{2}^{*} \\
U_{4} & 0 & -W_{2}^{*} \\
U_{5} & 0 & -W_{2}^{*}
\end{array}\right]\left[\begin{array}{c}
1 \\
U_{2} \\
U_{3}
\end{array}\right],
$$

where $U_{i}(i=1-5)$ are material invariants described in Ref. (13).

\subsection{Design of experiments and response surface method}

In the present study, flutter limit $\left(\lambda_{\mathrm{c}}^{*}\right)$ is approximated with a response surface. In this response surface, variables are the bending lamination parameters $\left(W_{1}^{*}, W_{2}^{*}\right)$. When the response is represented as $y$ and variables $W_{1}^{*}$ and $W_{2}^{*}$ are replaced with $x_{1}$ and $x_{2}$ for simplification, a response surface of a quadratic polynomial can be rewritten as

$$
y=\beta_{0}+\beta_{1} x_{1}+\beta_{2} x_{2}+\beta_{3} x_{1}^{2}+\beta_{4} x_{2}^{2}+\beta_{5} x_{1} x_{2} .
$$

The six unknown coefficients are calculated with the leastsquared error method from 12 sets of calculated responses and lamination parameters. Our previous study ${ }^{(9)}$ showed that the response surface obtained from the 12 sets provided sufficiently precise approximation for the buckling load problem. On the other hand, as mentioned before, the flutter problem usually has a sharp change of the flutter speed limit, which results from changes of vibration modes. This may create multiple local maxima. Our previous paper ${ }^{(12)}$ proposed a modified global response surface for panel flutter approximation. The present study employs the modified response surface to approximate the delta-wing flutter limit. In the modified response surface method, 15 sets, instead of 12 sets, are used for the leastsquared error method.

The additional three sets are not newly calculated laminates. The best laminate in the 12 sets is selected in the first step of this method. The selected best laminate is added as additional three sets to the 12 sets for the regression; then a response surface is recreated from the 15 $(=12+3)$ laminates. Although the method uses 15 sets for the regression, it requires only 12 sets for FEM analyses. This method reduces the bias only around the best laminate by adding three identical sets to the best laminate. In addition, this method is very effective for approximating the flutter limit, which has a sharp change of the response caused by the mode change.

Design of experiments with D-optimality is performed to reduce variance of the coefficients; 12 sets of the laminates are selected as shown in Fig. 2 from candidates of feasible laminates. Because the available fiber angles are limited to the small set of $0^{\circ}, \pm 45^{\circ}$, and $90^{\circ}$, the feasible space of the bending lamination parameter is bounded by the triangular domain of the three apexes: 


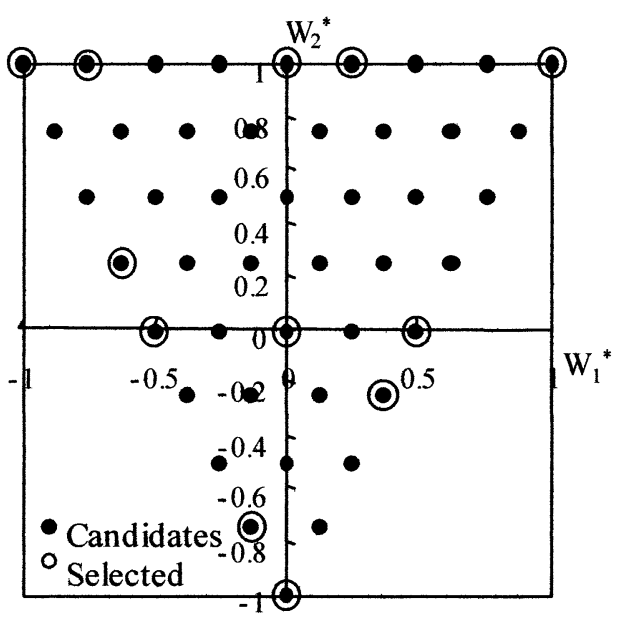

Fig. 2 Candidate points and selected points

$\left(W_{1}^{*}, W_{2}^{*}\right)=(-1,1),(1,1),(0,-1)$. The reason is described in detail in Ref.(6).

\subsection{Fractal branch and bound method}

Bending lamination parameters are expressed as progressions by counting the ply location from the outermost ply of a laminate as shown in Eq. (11). The values of $(\cos 2 \theta, \cos 4 \theta)$ in Eq. (11) are also limited to $0^{\circ}=(1,1)$, $\pm 45^{\circ}=(0,-1)$, and $90^{\circ}=(-1,1)$ because available fiber angles are limited to $0^{\circ}$-plies, $\pm 45^{\circ}$-plies, and $90^{\circ}$-plies, These values are identical to vertex vectors of the triangle of bending lamination parameters $\left(W_{1}^{*}, W_{2}^{*}\right)$ made from feasible laminates. All coefficients of the vectors of Eq. (11) are real numbers from 0 to 1 and bending lamination parameters of any stacking sequences are the linear sums of these three vectors. Thus, when all feasible laminates are plotted on bending lamination parameters, a fractal pattern is created inside and on the boundary of the triangle. Figure 3 shows the fractal pattern of half-ply number $N s=6$ (a symmetric laminate of total 12 plies) as an example.

In Fig. 3, the entire set of laminates that have a $0^{\circ}$ ply in the outermost ply is plotted in the shrunken triangle of the upper right. Correspondingly, the entire set of laminates that have $90^{\circ}$-ply in the outermost ply is plotted in the shrunken triangle of the upper left; the entire set of laminates that have a $45^{\circ}$-ply in the outermost ply is plotted in the shrunken lower triangle domain. When the second ply counted from outermost ply is decided, each shrunken triangle has other more shrunken triangles inside of each triangle. In this manner, with the decision of ply angles toward inside plies, this self-similar shrunken triangle is repeated inside each triangle to form the entire fractal pattern. Each triangle domain of laminates is called a fractal branch in the present study.

In the fractal branch and bound method, a branch and bound method is performed to obtain the optimal stackingsequence, which maximize a response surface of an ob-

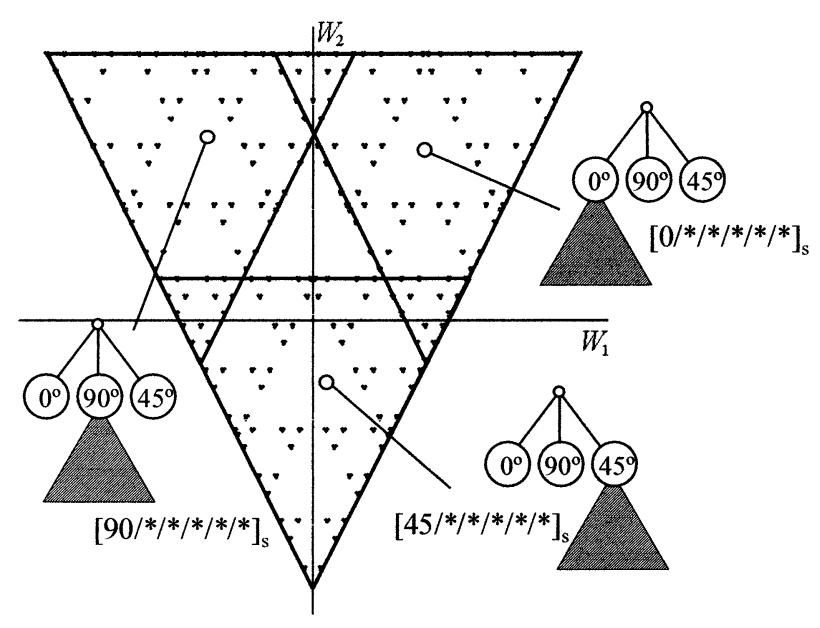

Fig. 3 Fractal structure of feasible laminates

jective function. A provisional optimal point $W_{\mathrm{op}}^{*}$ is obtained easily when we assume that lamination parameters are continuous real numbers using the response surface of the quadratic polynomial. However, for feasible laminates, available fiber angles are limited to a small set of fiber angles. This limitation makes the practical lamination parameters discrete numbers. The discrete number of the practical lamination parameters engenders a problem: a laminate that has exactly the same lamination parameters as the point of $W_{\mathrm{op}}^{*}$ does not always exist practically.

The branch and bound method for optimizing the stacking-sequence is performed to solve this problem. As mentioned previously, the provisional optimal point of $W_{\mathrm{op}}^{*}$ is obtained first. All feasible laminates located within the small domain are collected near the provisional optimal point. All feasible laminates in the domain around the provisional optimal are evaluated with the objective function of the response surface. The optimal stackingsequence that has the maximum value of the objective function is selected from the collection.

Fractal branches are used in the present study to collect the laminates around the provisional optimal point efficiently. Figure 4 shows this method schematically. In that figure, two shrunken triangles (fractal branches) intersect the domain around the provisional optimal point. Because the branches that have a $90^{\circ}$-ply in the outermost ply do not intersect with the domain around the provisional optimal point, as shown in Fig. 4, the fractal branch (a set of laminates) does not contain the optimal laminate. This allows pruning of the useless fractal branch. Lower hierarchies of the fractal branches are pruned similarly. In this manner, all feasible laminates within the domain near the provisional optimal point $W_{\mathrm{op}}^{*}$ are obtained. After obtaining the set of candidates around the optimal point $W_{\mathrm{op}}^{*}$, the actual optimal stacking-sequence can be selected by means of estimations of all candidate laminates using the response surface. This branch and bound 


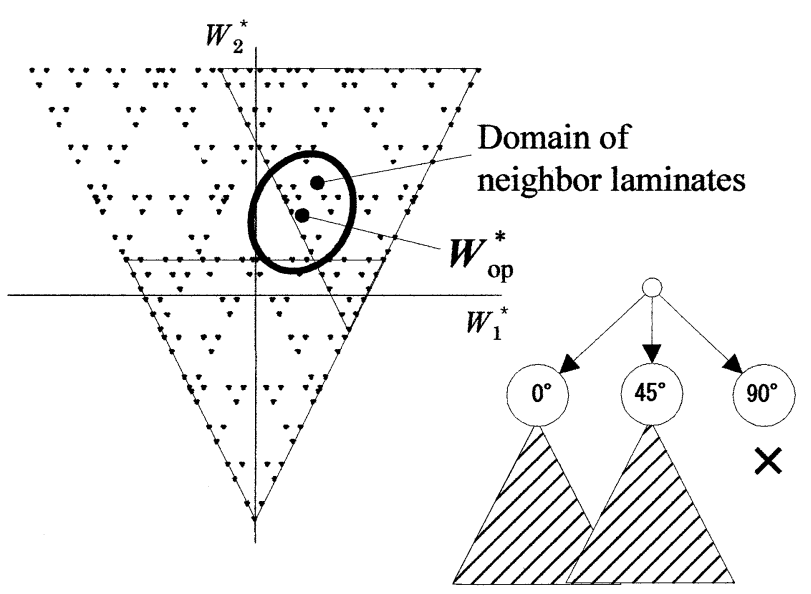

Fig. 4 Fractal structure and design space

method for optimizing the stacking-sequence of a laminate is named a fractal branch and bound method in the previous study ${ }^{(9)}$. Detailed description of the fractal branch and bound method is in Ref. (9).

\section{4 Flow of optimizations}

First, a response surface must be created to approximate the entire feasible lamination parameter $\left(W_{1}^{*}, W_{2}^{*}\right)$ space before stacking-sequence optimizations. In the present study, an objective function is the flutter limit $\lambda_{\mathrm{c}}^{*}$. The flutter limit $\lambda_{\mathrm{c}}^{*}$ comprises only bending stiffness of a laminate, as shown in Eq. (1). Therefore, only bending lamination parameters are regarded as variables in this optimization problem. Bending lamination parameters $W_{3}^{*}$ and $W_{4}^{*}$ are neglected here because the number of angle plies is balanced; also, a $+45^{\circ}$-ply is placed near the $-45^{\circ}$-ply here in the same manner mentioned in Ref. (5). To produce a precise approximation of the objective function with a response surface, design of experiments is performed from candidates of the entire set of feasible laminates with respect to bending lamination parameters $W_{1}^{*}$ and $W_{2}^{*}$. The design process of the experiments is described in Ref. (6). After selection of laminates for analyses, FEM analyses of the flutter limit are conducted. After FEM analysis, the calculated responses (flutter limit) of all selected laminates are used for obtaining coefficients of the response surface (quadratic polynomials) using the least-squared error method. This procedure is also listed in Ref. (6). After obtaining the response surfaces, the optimal laminate is sought using the fractal branch and bound method with the obtained response surface of flutter limit $\lambda_{\mathrm{c}}^{*}$.

\section{Result of Optimization and Discussion}

The fractal branch and bound method is adopted in this study for stacking-sequence optimization problems maximizing the flutter limit of the laminated delta wing. First, a flutter analysis is performed to obtain the flutter limit of a quasi-isotropic laminated delta wing for

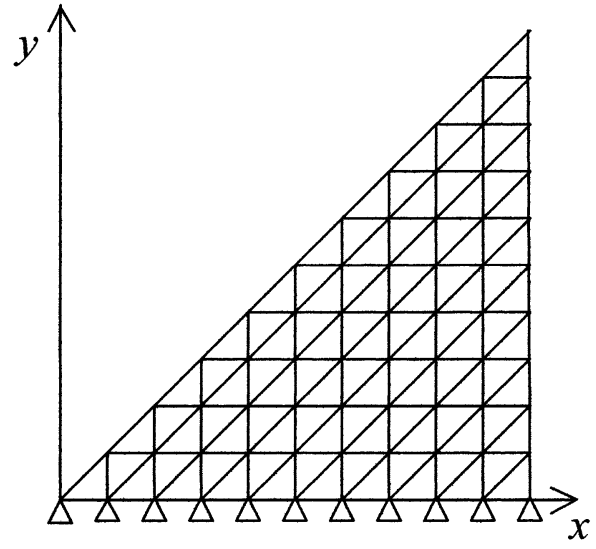

Fig. 5 Finite element assemblages for delta wing

comparison with the flutter limit of the optimal stackingsequence. Next, normal stacking-sequence optimization using a global modified response surface method is performed. Finally, stacking-sequence optimization is performed using a zoomed response surface to improve the optimization accuracy.

\subsection{Flutter analysis for quasi-isotropy}

An optimization model is a laminated composite delta wing, as shown in Fig. 1. Where, the ratio of the cord length $b$ to the span width $a$ is, $b / a=1$, and the wing thickness $h$ to the ratio of the span width $a$ is, $h / a=1.0 \times 10^{-2}$ respectively. It shows the flutter analysis result for quasiisotropic. The flutter limit is analyzed using finite element method. The delta wing, Fig. 1, is divided into an $11 \times 11$ mesh, 100 elements, giving 165 degrees of freedom, as shown in Fig. 5.

In Eq. (9), eigenvalues are calculated by changing the Mach number $M$, i.e. $\lambda$. Figure 6 shows the result of the flutter analysis. Flutter limit $\lambda_{\mathrm{c}}$ is the point at which the two different eigenvalue modes coincide with each other first. The obtained flutter limit is normalized as Eq. (10). The resultant flutter limit in the quasi-isotropic laminated delta wing, the value of $\lambda_{\mathrm{c}}^{*}$, is 69.18 .

\section{2 Optimization result for global modified re- sponse surface}

As described previously, Fig. 1 shows an optimization model for a laminated composite delta wing. The stacking-sequence of the laminate is optimized to maximize the flutter speed limit using the fractal branch and bound method. The geometrical configuration of this optimization model is the same as the configuration used in the section 4.1; the ratio of the cord length $b$ to the span width $a$ is, $b / a=1$, and the wing thickness $h$ to the ratio of the span width $a$ is, $h / a=1.0 \times 10^{-2}$ respectively.

As described previously, in the first step, a response surface of flutter limit must be created to approximate the entire feasible lamination parameter space $\left(W_{1}^{*}, W_{2}^{*}\right)$. Design of experiments with D-optimality is performed to obtain a precise approximation of the objective function with 


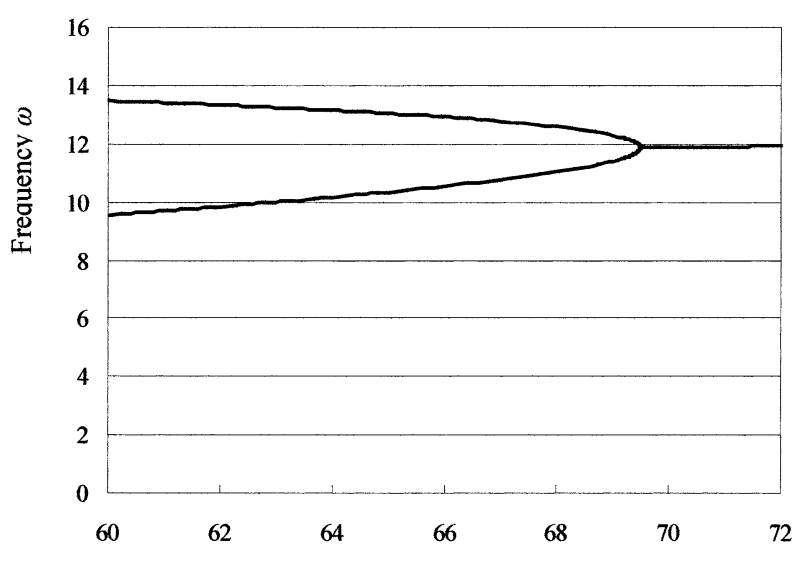

Flutter parameter $\lambda^{*}$

Fig. 6 Result of the flutter analysis for quasi-isotropic

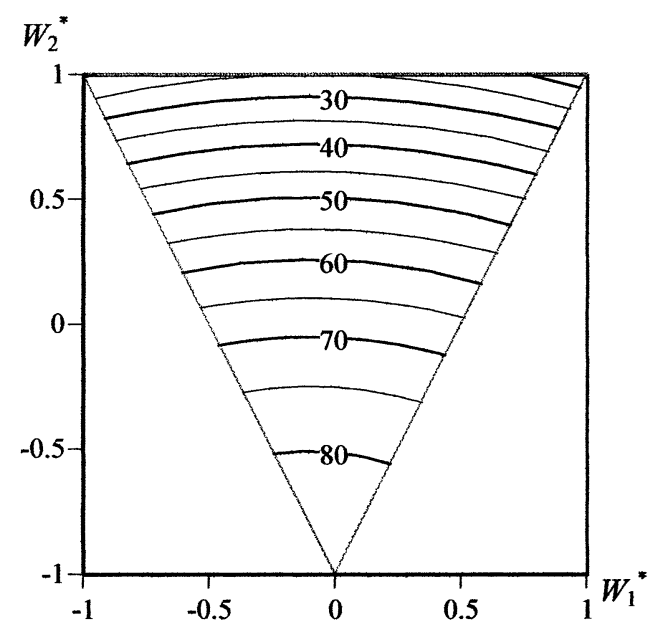

Fig. 7 Contour plot of response surface of flutter speed limit $\lambda_{\mathrm{c}}^{*}$

a response surface; thereupon, as shown in Fig. 2, 12 sets of laminates are selected from candidates of feasible laminates. However, the origin point is surely included in the D-optimality design of the experiment to reduce bias around the origin point.

Flutter analyses are performed in all 12 selected laminates. As described previously, the flutter speed limit is analyzed using finite element method. The delta wing, as in Fig. 1, is divided into an $11 \times 11$ mesh with 100 elements, giving 165 degrees of freedom, as shown in Fig. 5. A response surface is created with the modified response surface method using obtained flutter analysis results for the 12 laminates; 15 data are used for making the response surface. The obtained response surface is shown as

$$
\lambda_{\mathrm{c}}^{*}=68.52-1.4 W_{1}^{*}-29.52 W_{2}^{*}-7.05 W_{1}^{* 2}-13.85 W_{2}^{* 2},
$$

where $R_{\text {adj }}^{2}=0.9932$. Figure 7 shows the contour plot of the obtained response surface.

The overall response of the bending lamination pa-

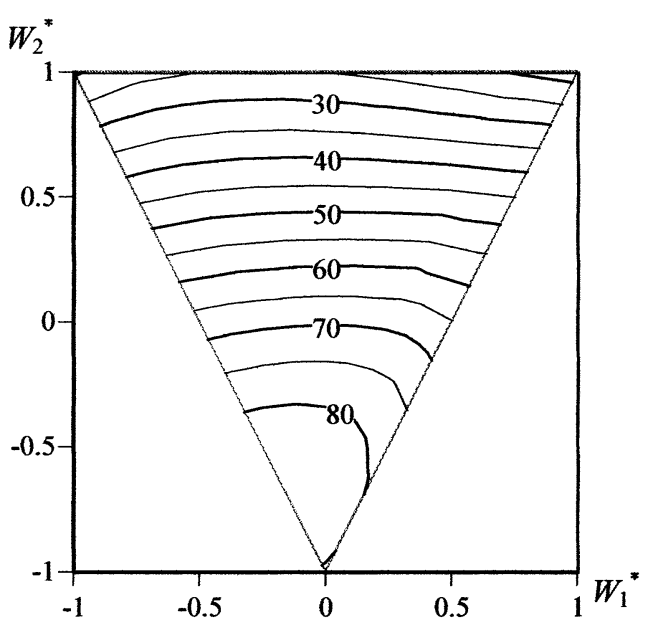

Fig. 8 Contour plots of flutter speed limit $\lambda_{\mathrm{c}}^{*}$

rameters, practical design space, is obtained here to confirm optimality. Figure 8 shows this contour plot. Of course, these analyses of the entire design space are very time consuming; moreover, they are not acceptable for practical design. When both are compared, the obtained modified response surface represents good approximation of practical design space in outline.

The fractal branch and bound method is performed to obtain the response surface [Eq. (14)], and an optimal stacking-sequence to maximize flutter speed limit is obtained. This study addresses stacking-sequence optimization in a symmetric laminate of a total of 12 plies. The obtained optimal stacking-sequence is $\left[(45 /-45)_{3}\right]_{\mathrm{s}}$. The coordinate in the bending lamination parameters space of this obtained optimal laminate is $\left(W_{1}^{*}, W_{2}^{*}\right)=(0,-1)$; its value of $\lambda_{\mathrm{c}}^{*}$ is 79.18. Adjacent laminates to the optimal laminate are analyzed. The true optimal laminate is [45/ $-45 / 90 / 45 /-45 / 0]_{\mathrm{s}}$; the coordinate in the bending lamination parameters space is $\left(W_{1}^{*}, W_{2}^{*}\right)=(-0.167,-0.648)$; the value of $\lambda_{\mathrm{c}}^{*}$ is 84.86 . The error of the flutter speed limit against the true optimal laminate is less than $6.7 \%$. This confirms that the obtained optimal laminate is practically acceptable.

However, the optimization accuracy is insufficient compared with the problem of maximizing of panel flutter limit ${ }^{(12)}$. In stacking-sequence optimization using the fractal branch and bound method, the approximated coordinate of the maximum point in the bending lamination parameter space is more important than the absolute value of the approximated flutter limit of the optimal laminate. Figures 6 and 7 show that a slight difference exists between the results of the two figures in the domain near the maximum point. The standard approach for improving the accuracy of a global response surface is zooming. This zooming requires a zoomed response surface near the optimum point obtained from the global response surface. Then the next step is performed: stacking-sequence opti- 


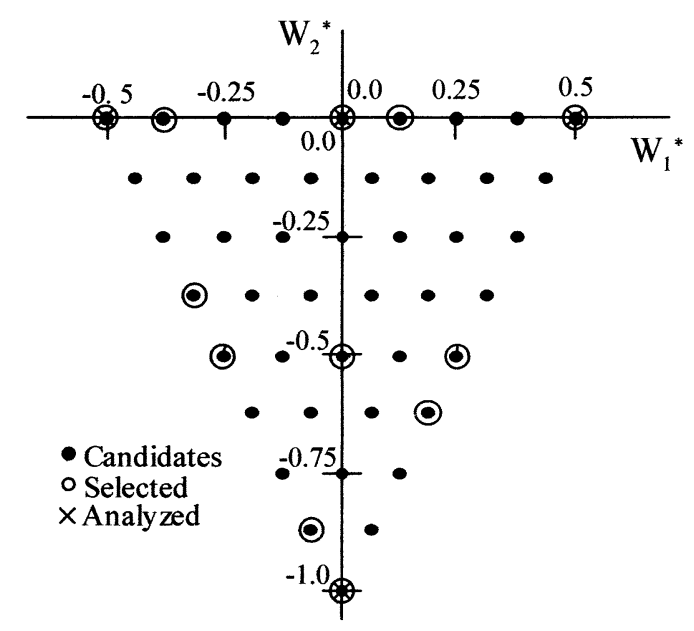

Fig. 9 Candidate points and selected points for zoomed response surface

mization using the zoomed response surface.

\subsection{Optimization result for zoomed modified re- sponse surface}

Stacking-sequence optimization for the zoomed modified response surface using the fractal branch and bound method is performed to improve accuracy of the optimization result. In this optimization, the treated analysis model is the same as the section 4.2. The zoomed response surface is constructed in the region $W_{2}^{*} \leq 0$ and $W_{2}^{*} \geq-2 W_{1}^{*}-1$. In this region design of experiments, D-optimality is performed again to precisely approximate the objective function with a response surface; as shown in Fig. 9, 12 sets of laminates are selected again in this zoomed area from the candidates of feasible laminates. Flutter analyses are performed for all 12 selected laminates. Here, there are eight newly selected points; four points are already analyzed to make the global response surface. Therefore, eight analysis iterations are required to produce the zoomed response surface. The flutter speed limit is analyzed using the finite element method, as in the section 4.2. Using obtained flutter analysis results for the 12 laminates (four analyzed previously and eight newly analyzed), a response surface is created with the modified response surface method: 15 data are used for producing the response surface. The obtained response surface is explained by the following equation:

$$
\begin{aligned}
\lambda_{\mathrm{c}}^{*} & =38.73-4.58 W_{1}^{*}-29.76 W_{2}^{*} \\
& -6.28 W_{1}^{* 2}+4.99 W_{1}^{*} W_{2}^{*}-15.38 W_{2}^{* 2},
\end{aligned}
$$

where $R_{\mathrm{adj}}^{2}=0.9937$. Figure 10 shows the contour plot of the obtained zoomed response surface.

The zoomed response surface is compared with the true response in the bending lamination parameters to confirm the optimality (Fig. 8). As a result, the zoomed response surface represents good approximation of practical design space near the maximum point.

The fractal branch and bound method used the

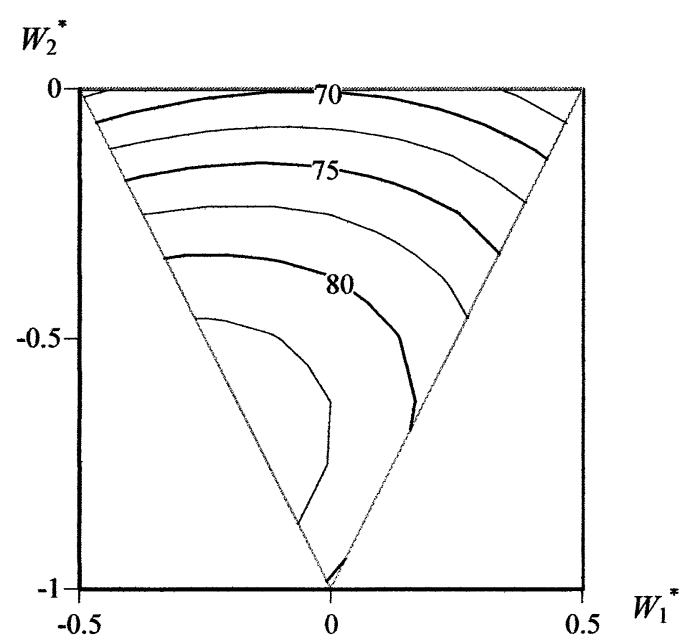

Fig. 10 Contour plot of zoomed response surface for flutter speed limit $\lambda_{\mathrm{c}}^{*}$

zoomed response surface [Eq. (15)] to obtain the optimal stacking-sequence in a symmetric laminate of 12 plies. This solution maximizes the flutter speed limit. The obtained optimal stacking-sequence is $\left[(45 /-45 / 90)_{2}\right]_{s}$. The coordinate in the bending lamination parameter space of this obtained optimal laminate is $\left(W_{1}^{*}, W_{2}^{*}\right)=$ $(-0.176,-0.648)$; the value of $\lambda_{\mathrm{c}}^{*}$ is 84.26 . As described previously, the true optimal laminate is $[45 /-45 / 90 / 45 /-$ $45 / 0]_{\mathrm{S}}$ and the coordinate in the bending lamination parameter space is $\left(W_{1}^{*}, W_{2}^{*}\right)=(-0.167,-0.648)$; the value of $\lambda_{\mathrm{c}}^{*}$ is 84.86 . The error of the flutter speed limit against the true optimal laminate is less than $0.3 \%$. We confirmed that use of the zoomed response surface the radically improved the accuracy of the optimal stacking-sequence. The obtained optimal laminate is practically acceptable. The obtained flutter limit of optimal stacking-sequence was then compared with flutter limit of quasi-isotropic stackingsequence to confirm optimality. Consequently, performing stacking-sequence optimization confirmed that the flutter limit is improved by $22 \%$ or more.

\section{Conclusions}

The fractal branch and bound method was applied to stacking-sequence optimization of composite laminates. The objective function must be approximated precisely with a quadratic-polynomial response surface in the bending lamination parameter space to perform the fractal branch and bound method for stacking-sequence optimizations of laminated composites.

In this study, the fractal branch and bound method was applied to the stacking-sequence optimization problem to maximize the flutter speed limit of a composite delta wing. A practical optimal stacking-sequence was obtained. In addition, the feasibility of this method was confirmed. Conclusions obtained in this study are the following: 
( 1 ) In stacking-sequence optimization for maximizing the flutter limit of a composite delta wing, the flutter limit design space can be approximated excellently using the modified response surface method; the optimal stacking-sequence is obtained using the fractal branch and bound method. The proposed method requires a low computational cost.

(2) Stacking-sequence optimization for a zoomed response surface is performed to improve optimal accuracy. It is confirmed that the stacking-sequence optimization for the zoomed response surface provided a practically optimal laminate in high accuracy. Only eight additional FEM analyses are needed for this optimization.

\section{Acknowledgement}

The authors are grateful to the advice for flutter analyses of professor H. Furuya of Tokyo Institute of Technology.

\section{References}

(1) Miki, M., Design of Laminated Fibrous Composite Plates with Required Flexural Stiffness, ASTM, STP, Vol.864 (1985), pp.387-400.

( 2 ) Fukunaga, H. and Chou, T.W., Simplified Design Techniques for Laminated Cylindrical Pressure Vessels under Stiffness and Strength Constraints, J. of Composite Materials, Vol.22 (1998), pp.1156-1169.

( 3 ) Todoroki, A. and Haftka, R.T., Stacking Sequence Matching Using Genetic Algorithm with Repair, Composites Part B, Vol.29, No.8 (1998), pp.277-285.

(4) Todoroki, A. and Haftka, R.T., Stacking Sequence Matching by Two Stage Genetic Algorithm with Consanguineous Initial Population, AIAA Paper 97-1228, Proceedings, 38th AIAA/ASME/ASCE/AHS/ASC Structures and, Structural Dynamics, and Materials Conference and Exhibit and AIAA/ASME/AHS Adaptive Structures Forum, Kissimmee, FL, (1997), pp.1297-1302.

( 5 ) Todoroki, A. and Sasai, M., Improvement of Design Reliability for Buckling Load Maximization of Composite Cylinder Using Genetic Algorithm with Recessive-Gene-Like Repair, JSME Int. J., Ser. A, Vol.42, No.4 (1999), pp.530-536.

( 6 ) Todoroki, A. and Haftka, R.T., Lamination Parameters for Efficient Genetic Optimization of the Stacking Se- quences of Composite Panels, AIAA Paper 98-4817, Proceedings, 7th AIAA/USAF/NASA/ISSMO Symposium on Multidisciplinary Analysis and Optimization, St. Louis, MO, (1998), pp.870-879.

( 7 ) Todoroki, A. and Ishikawa, T., Design of Experiments for Response Surfaces of Stacking Sequence Optimizations Using Genetic Algorithm, Trans. Jpn. Soc. Mech. Eng., (in Japanese), Vol.66, No.645, A (2000), pp.978985.

( 8 ) Todoroki, A. and Sasai, M., Stacking Sequence Optimizations Using GA with Zoomed Response Surface on Lamination Parameters, Advanced Composite Materials, Vol.11, No.3 (2003), pp.299-318.

( 9 ) Terada, Y., Todoroki, A. and Shimamura, Y., Stacking Sequence Optimizations Using Fractal Branch and Bound Method for Laminated Composites, JSME Int. J., Ser. A, Vol.44, No.4 (2001), pp.490-498.

(10) Kameyama, M., Ozeki, T., Fukunaga, H., Nakamichi, J. and Sekine, H., Research on Optimum Design of Composite Late Wings for Aeroelastic Characteristics Using Lamination Parameters (The First Report) Flutter and Divergence Characteristics of Composite Plate Wings - J. of the Japan Society for Aeronautical and Space Sciences, (in Japanese), Vol.51, No.595 (2003) pp.441-448.

(11) Satou, M., Fukunaga, H. and Sekine, H., Panel Flutter Design of Symmetrically Laminated Plates Using Lamination Parameters, Trans. Jpn. Soc. Mech. Eng., (in Japanese), Ser. C, Vol.64, No.619 (1998), pp.10131020.

(12) Todoroki, A. and Hirano, Y., Stacking Sequence Optimizations of Composites to Improve Panel Flutter at Supersonic Mach Numbers Using Fractal Branch and Bound Method, Trans. Jpn. Soc. Mech. Eng., (in Japanese), Vol.69, No.678, A (2003), pp.239-244.

(13) Gürdal, Z., Haftka, R.T. and Hajela, P., Design and Optimization of Laminated Composite Materials, (1999), John Wiley \& Sons, Inc., New York.

(14) Ashley, H. and Zartarian, G., Piston Theory - A New Aerodynamic Tool for the Aeroelastician, J. of Aero. and Sci., Vol.23, No.12 (1956), pp.1109-1118.

(15) Zienkiexicz, O.C., The Finite Element Method, (1978), McGraw-Hill Book Company, UK.

(16) Wilkinson, J.H. and Reinsch, C., Linear Algebra, Vol. II of Handbook for Automatic Computation, (1971), Springer-Verlag, New York. 